\title{
Denoising-based Image Compression for Connectomics
}

\author{
David Minnen $^{1, \#}$, Michał Januszewski ${ }^{1, \#}$, Alexander Shapson-Coe ${ }^{2}$, \\ Richard L. Schalek², Johannes Ballé ${ }^{1}$, Jeff W. Lichtman², Viren Jain ${ }^{1}$ \\ ${ }^{2}$ Department of Molecular and Cellular Biology \& Center for Brain Science, Harvard University
}

${ }^{1}$ Google Research

*Equal contribution

\begin{abstract}
Connectomic reconstruction of neural circuits relies on nanometer resolution microscopy which produces on the order of a petabyte of imagery for each cubic millimeter of brain tissue. The cost of storing such data is a significant barrier to broadening the use of connectomic approaches and scaling to even larger volumes. We present an image compression approach that uses machine learning-based denoising and standard image codecs to compress raw electron microscopy imagery of neuropil up to 17 -fold with negligible loss of reconstruction accuracy.
\end{abstract}

\section{Main Text}

Progress in the reconstruction and analysis of neural circuits has recently been accelerated by advances in 3d volume electron microscopy (EM) and automated methods for neuron segmentation and annotation ${ }^{1,2}$. In particular, contemporary serial section EM approaches are capable of imaging hundreds of megapixels per second ${ }^{3,4}$, which has led to the successful acquisition of petabyte-scale volumes of human ${ }^{5}$ and mouse ${ }^{6}$ cortex. However, scaling further poses substantial challenges to computational infrastructure; nanometer resolution imaging of a whole mouse brain ${ }^{7}$ could, for example, produce one million terabytes (an exabyte) of data. While storage systems with such capacities are available, they are costly to build and operate. More generally, even petabyte-scale requirements limit the number and diversity of circuit reconstruction efforts. Thus in order to mitigate the costly storage demands of connectomics, we developed a practical approach to compression of electron microscopy data that yields at least a 17 -fold reduction in storage requirements.

The key insight is that computational removal of imaging noise allows EM images to be compressed far more effectively than in their raw form ${ }^{8,9}$. Since an effective noise model for complex microscopy images can be difficult to formulate a priori, we instead learn a denoising model directly from EM imagery itself. We acquired pairs of EM scans of human cortical tissue ${ }^{5}$ under different imaging speeds of a Zeiss 61-beam scanning electron microscope ${ }^{3}$, which resulted in images of the same tissue but containing different amounts of noise (i.e., fast with more noise vs slow with less noise). After precise alignment of fast and slow scans (see Methods), the image pairs were used to train a dense prediction neural network ${ }^{10}$ with standard supervised techniques. The trained network was then used to infer "slow" 3200 nanosecond 
low-noise scans (Figure 1) from the "fast" 200 nanosecond acquisition scans (imaging all data in the low-noise regime would be too slow for large tissue volumes).

Denoised EM imagery was then compressed using a lossy codec (JPEG XL and AVIF). These codecs provide tunable parameters that trade off compression rate for image fidelity. Beyond visual inspection (Figure 2) we wanted to establish that automated reconstruction of the data would not be degraded by denoising or compression. Therefore we used flood-filling networks (FFNs) ${ }^{11}$ to segment neurons based on original, compressed, and denoised+compressed data and compared the reconstruction results to human ground truth.

We found that denoising alone did not impact reconstruction and that compression of denoised data enabled 17-fold storage reduction with negligible loss of reconstruction accuracy according to an "edge accuracy" metric that quantified the number of splits and mergers in an automated result ${ }^{11}$ (Figure 3 ). This compression rate was more than $3 \mathrm{x}$ better compared to what was achievable with data that was compressed without first being denoised.

We believe the proposed approach is a highly practical method of achieving compression in real-world connectomics pipelines, for several reasons. First, training the denoising model requires an insignificant amount of additional imaging relative to an overall large-scale acquisition effort. For instance, in our experiments we used tens of gigabytes of images acquired at slow and fast acquisition speeds, which was equivalent to less than $0.005 \%$ of the complete dataset. Second, both the denoising and compression codecs operate on $2 \mathrm{~d}$ data which means the models can be applied to unstitched and unaligned raw data directly from the microscope. Third, denoising involves inference with a relatively standard U-Net neural network architecture for which inference can be computed at tens of megapixels per second on a single GPU ${ }^{12}$; compression involves codecs such as JPEG XL which also can be computed at tens of megapixels per second on a single $\mathrm{CPU}^{13}$.

Finally, we note that our approach does not rely on a learned compression model ${ }^{14}$. This simplifies implementation and enables a flexible approach to choosing compression parameters. That said, learned compression remains an interesting avenue for potential further reductions in storage requirements. The application of volumetric learned compression models ${ }^{15}$ to post-aligned EM data is also an interesting research direction. However, the approach described here already reduces storage requirements by an order of magnitude and thus dramatically increases feasibility of existing and proposed ${ }^{7}$ projects. 


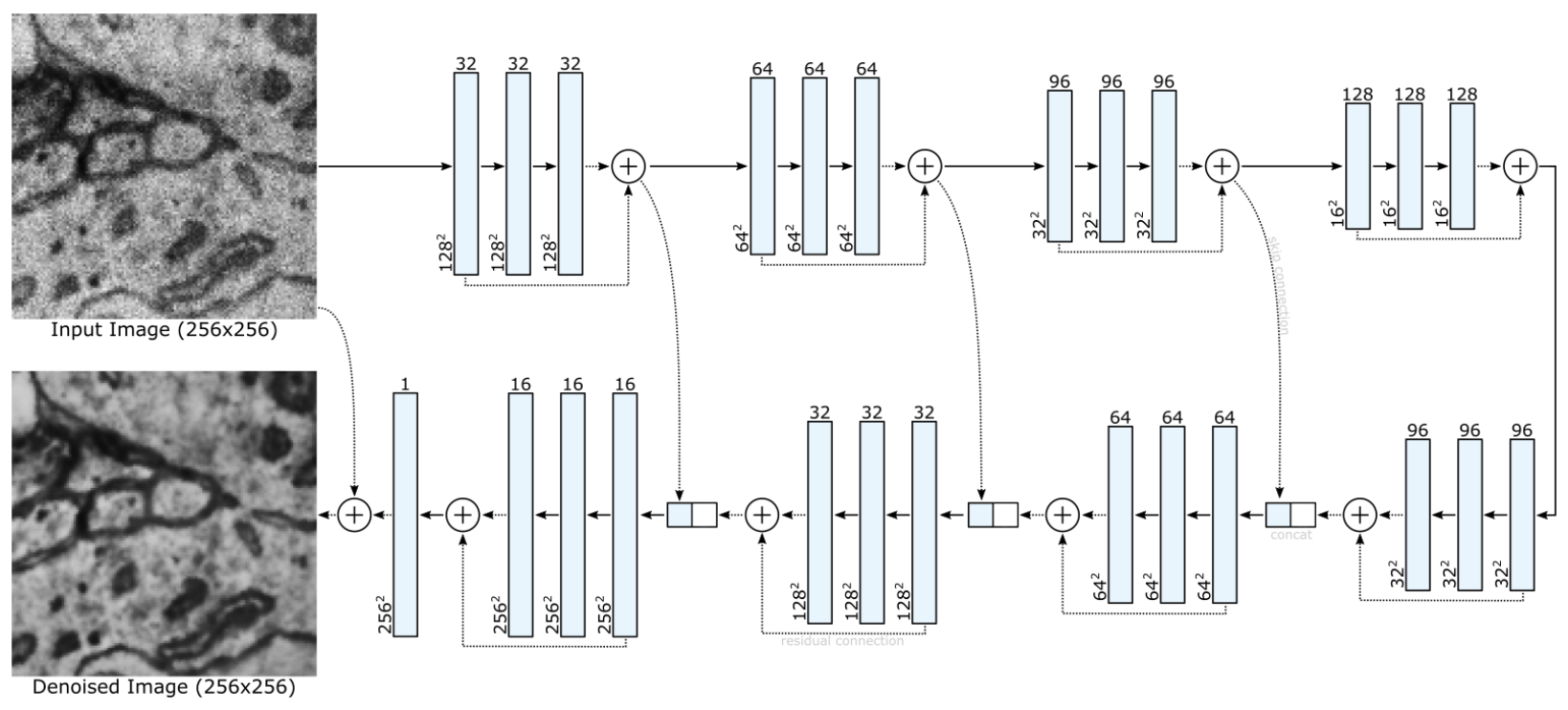

Figure 1. A deep neural network learns to denoise tissue scans after being optimized on aligned pairs of high-noise (fast dwell time) and low-noise (slow dwell time) scans. The network uses a U-Net architecture ${ }^{10}$ with skip connections between the downscaling and upscaling paths. Residual connections are also used for both the individual blocks and for the final output.

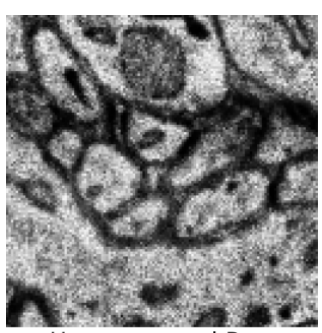
Uncompressed Raw

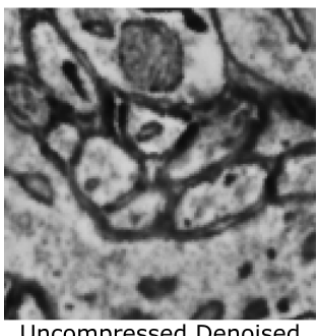

Uncompressed Denoised

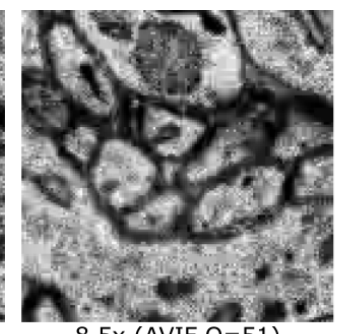

$8.5 \times(A V I F \mathrm{Q}=51)$

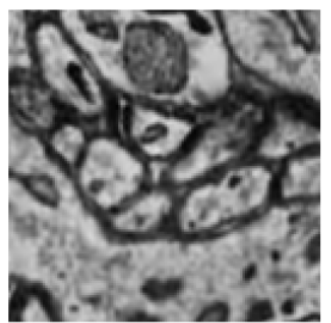

$10.25 \times($ AVIF $Q=36)$

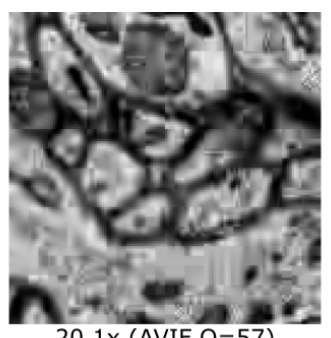

$20.1 \times($ AVIF $Q=57)$

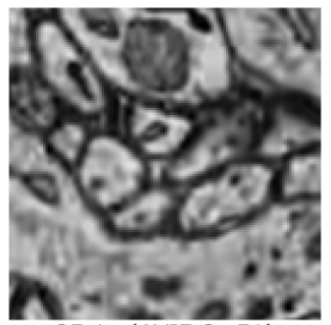

$25.1 \times(\operatorname{AVIF} Q=51)$

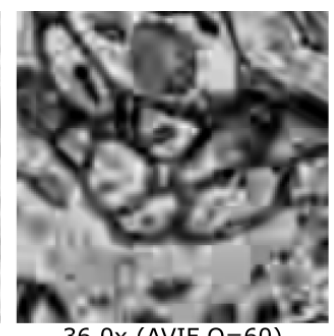

36.0x (AVIF $Q=60)$

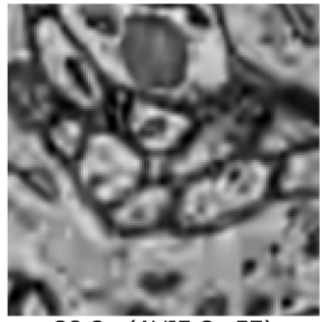

$38.2 \times($ AVIF $Q=57)$
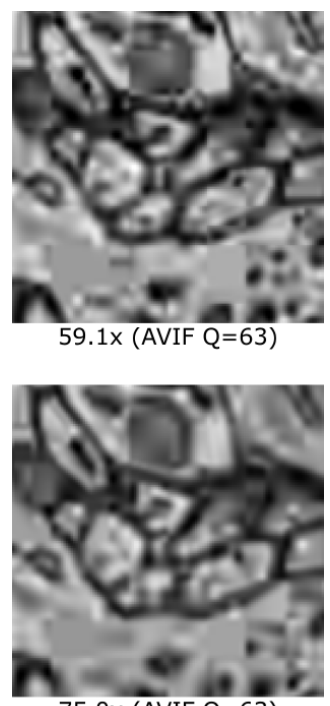

75.0x (AVIF Q=63)

Figure 2. By first denoising the raw data, both compression rates and visual quality increase (bottom row) compared to directly compressing the noisy scans (top row). Specifically, fewer blocking artifacts (due to the block-based partition used by AVIF) and fewer geometric artifacts (due to the discrete cosine transform) appear in the compressed version of the denoised scans. Online side-by-side views at comparable compression rates are available for $\underline{\text { VVIF }}$ and $\underline{\mathrm{JXL}}$. 


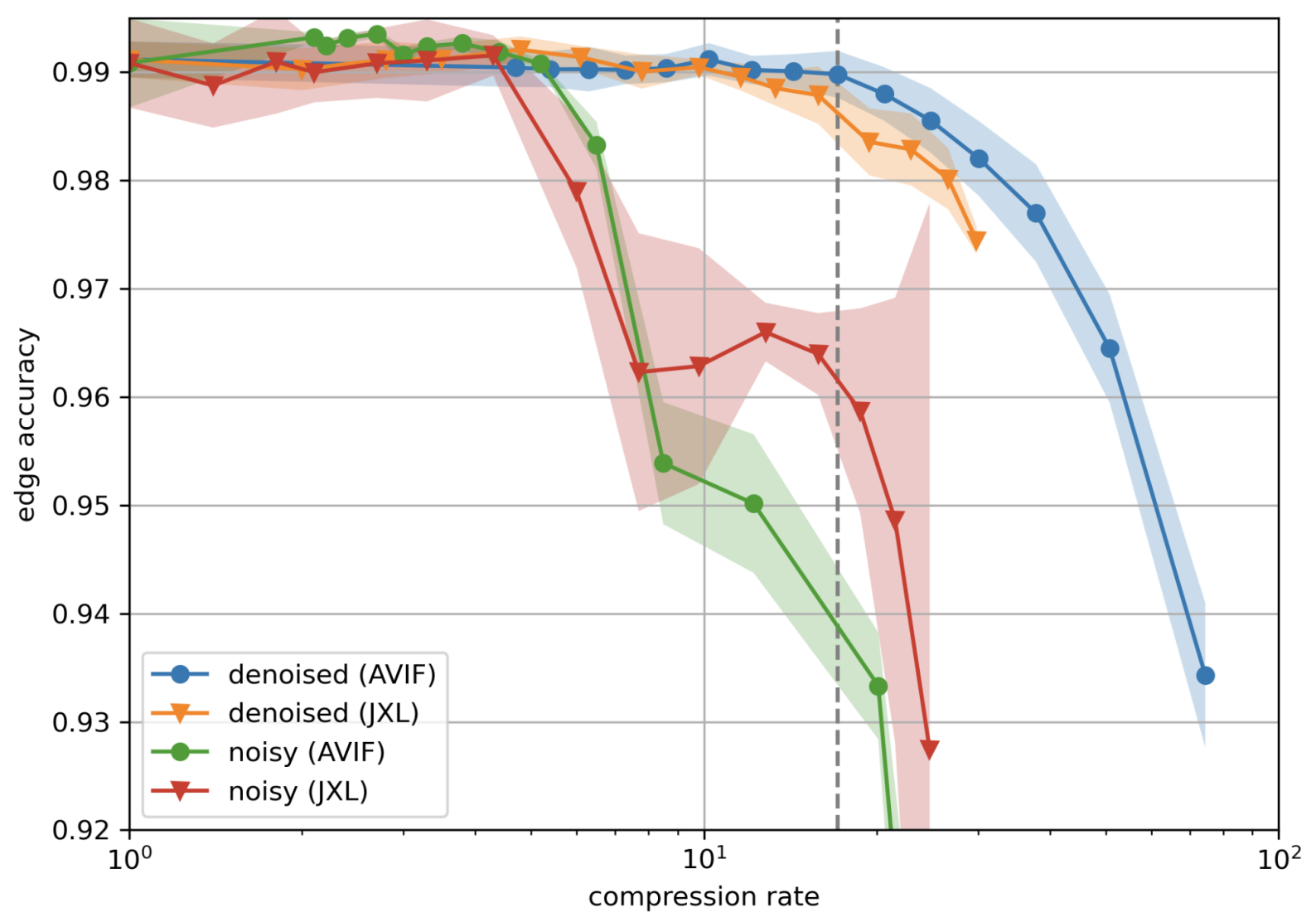

Figure 3. Segmentation quality of noisy and denoised images as a function of compression rate. Points and lines show the means, and the shaded area covers \pm 1 standard deviation around the mean. Statistics were computed over segmentations with 5 different $8 \times 8 \times 30 \mathrm{~nm}^{3}$ FFN network checkpoints. The dashed line corresponds to a $17.1 \mathrm{x}$ compression rate. Compression rates are computed with $8 \times 8 \times 30 \mathrm{~nm}^{3}$ voxel volume as the baseline, which was the highest resolution used for automated processing. Taking downsampling into account, $17.1 \mathrm{x}$ therefore corresponds to a $68.4 x$ size reduction relative to the size of images as originally acquired with $4 \times 4 \mathrm{~nm}^{2}$ pixels. 


\section{References}

1. Kornfeld, J. \& Denk, W. Progress and remaining challenges in high-throughput volume electron microscopy. Curr. Opin. Neurobiol. 50, 261-267 (2018).

2. Lee, K. et al. Convolutional nets for reconstructing neural circuits from brain images acquired by serial section electron microscopy. Current Opinion in Neurobiology vol. 55 188-198 (2019).

3. Eberle, A. L. et al. High-resolution, high-throughput imaging with a multibeam scanning electron microscope. J. Microsc. 259, 114-120 (2015).

4. Robinson, C. G. et al. Automated Infrastructure for High-Throughput Acquisition of Serial Section TEM Image Volumes. Microscopy and Microanalysis vol. 22 1150-1151 (2016).

5. Shapson-Coe, A. et al. A connectomic study of a petascale fragment of human cerebral cortex. (2021)

6. Yin, W. et al. A petascale automated imaging pipeline for mapping neuronal circuits with high-throughput transmission electron microscopy. Nat. Commun. 11, 4949 (2020).

7. Abbott, L. F. et al. The Mind of a Mouse. Cell 182, 1372-1376 (2020).

8. Oh, B. T., Kuo, C.-C. J., Sun, S. \& Lei, S. Film grain noise modeling in advanced video coding. Visual Communications and Image Processing 2007 (2007) doi:10.1117/12.707808.

9. Dai, J., Au, O. C., Pang, C., Yang, W. \& Zou, F. Film grain noise removal and synthesis in video coding. 2010 IEEE International Conference on Acoustics, Speech and Signal Processing (2010) doi:10.1109/icassp.2010.5495254.

10. Ronneberger, O., Fischer, P. \& Brox, T. U-net: Convolutional networks for biomedical image segmentation. in International Conference on Medical Image Computing and Computer-Assisted Intervention 234-241 (Springer, 2015).

11. Januszewski, M. et al. High-precision automated reconstruction of neurons with flood-filling 
networks. Nat. Methods 15, 605-610 (2018).

12. NVIDIA NGC.

https://ngc.nvidia.com/catalog/resources/nvidia:unet_medical_for_tensorflow/performance.

13. Alakuijala, J. et al. Benchmarking JPEG XL image compression. Optics, Photonics and Digital Technologies for Imaging Applications VI (2020) doi:10.1117/12.2556264.

14. Ballé, J., Laparra, V. \& Simoncelli, E. P. End-to-end Optimized Image Compression. (2017).

15. Agustsson, E. et al. Scale-Space Flow for End-to-End Optimized Video Compression. 2020 IEEE/CVF Conference on Computer Vision and Pattern Recognition (CVPR) (2020) doi:10.1109/cvpr42600.2020.00853.

16. Saalfeld, S., Fetter, R., Cardona, A. \& Tomancak, P. Elastic volume reconstruction from series of ultra-thin microscopy sections. Nature Methods vol. 9 717-720 (2012).

17. Zach, C., Pock, T. \& Bischof, H. A Duality Based Approach for Realtime TV-L 1 Optical Flow. Lecture Notes in Computer Science 214-223 doi:10.1007/978-3-540-74936-3_22.

18. Hendrycks, D. \& Gimpel, K. Gaussian Error Linear Units (GELUs). arXiv [cs.LG] (2016).

19. Wang, Z., Bovik, A. C., Sheikh, H. R. \& Simoncelli, E. P. Image Quality Assessment: From Error Visibility to Structural Similarity. IEEE Transactions on Image Processing vol. 13 600-612 (2004).

20. Zhang, R., Isola, P., Efros, A. A., Shechtman, E. \& Wang, O. The Unreasonable Effectiveness of Deep Features as a Perceptual Metric. 2018 IEEE/CVF Conference on Computer Vision and Pattern Recognition (2018) doi:10.1109/cvpr.2018.00068.

21. Kingma, D. P. \& Ba, J. Adam: A method for stochastic optimization. arXiv preprint arXiv:1412.6980 (2014). 


\section{Supplementary Methods}

\section{Electron Microscopy Data}

We used a small subset of the $1 \mathrm{~mm}^{3}$ human cortex $\mathrm{H} 01$ sample $^{5}$ for all experiments. The surgical tissue was extracted from the left medial temporal lobe of a 45-year old woman with a history of seizures, and fixed immediately after excision. The excised sample was free of gross pathology. It was subsequently stained using the ROTO protocol, embedded in resin, and sectioned with an ATUM device. The part of the sample we used was cut with a section thickness of $30 \mathrm{~nm}$. The sections were affixed to silicon wafers and imaged with a 61-beam Zeiss MultiSEM microscope at a pixel resolution of $4 \times 4 \mathrm{~nm}^{2}$, and with a beam dwell time of $200 \mathrm{~ns}$, current of $576 \mathrm{pA}$, and landing energy of $1.5 \mathrm{kV}$.

The image tiles were stitched and aligned by Adi Peleg using a custom software implementation of elastic alignment ${ }^{16}$. We normalized the contrast of the aligned volume with contrast limited adaptive histogram equalization (CLAHE) applied grid-wise within 1300x1300-pixel patches with an overlap of $300 \times 300$ pixels.

\section{Scan Registration}

Training data is extracted from two sets of scans of the same tissue, one at $200 \mathrm{~ns}$ and one at 3200 ns. The corresponding images between the two scans have considerable overlap but are not precisely aligned as captured. To achieve pixel-accurate alignment, each $3200 \mathrm{~ns}$ image is processed by a four-stage pipeline. First, all images are normalized using CLAHE. Second, each $3200 \mathrm{~ns}$ image is roughly aligned to its $200 \mathrm{~ns}$ counterpart via translation by estimating the $2 \mathrm{~d}$ offset using cross-correlation. The grayscale pixel values are then adjusted to better match the 200 ns image by solving a linear regression problem, and, finally, the image undergoes nonrigid warping according to a flow field estimated by multiscale TV-L1 optical flow ${ }^{17}$.

\section{Denoising}

The denoising model works by learning to map noisy scans captured using 200 ns dwell times to low-noise scans captured using $3200 \mathrm{~ns}$ dwell times. The network architecture follows the U-Net approach ${ }^{10}$, where input images are analyzed at successively lower resolutions down one path and then mapped to output images via upscaling on a second path (Figure 1). Skip connections between the two paths allow the network to more easily utilize high-resolution information, while residual connections are used both within each block in the U-Net and for the final output.

Our model uses U-Net blocks with three convolutional layers. The first uses a stride of two with $5 \times 5$ kernels, while the second and third layers use a stride of one with $3 \times 3$ kernels. In the upscaling path, the stride two convolutions are replaced with transposed convolution to increase, rather than decrease, the spatial resolution, and all layers use "same" padding. Gaussian Error Linear Units (GELUs) ${ }^{18}$ are used between each convolutional layer. 
Four residual blocks are used in the downscaling path to reduce the resolution by a total of $16 x$. The blocks increase the channel depth from a single grayscale input image to $32,64,96$, and finally 128 channels. The upscaling path mirrors this structure and uses depths of 96, 64, 32, and 16 for its blocks. A single final convolution using a $3 \times 3$ kernel with an output depth of one generates a grayscale residual. The denoised image is then produced by adding this residual to the input image.

Training makes use of $256 \times 256$ patch pairs extracted from the aligned scans. The network is fully convolutional so the input resolution is not explicitly constrained by the architecture, but limited memory on real hardware requires us to tile the input for very large images. When denoising on a CPU, we used 4096x4096 tiles, but smaller tiles may be required for GPUs with less onboard memory. To minimize boundary artifacts, we used an additional border of 128 pixels on all sides that is dropped after denoising. In the case where 128 extra pixels are not available, e.g., at the edge of the scan volume, reflection-based padding is used.

To drive the optimization process, a differentiable loss function is needed. We don't expect perfect prediction of the low-noise scans, and so, ideally, the loss function would optimally score image discrepancies in proportion to their effect on downstream analysis both by scientists and by automated systems. In theory, we could use the segmentation algorithm as a loss function, but it is currently a multi-stage process that is not fully differentiable. Regardless, even if we could train in an end-to-end fashion with the segmentation algorithm, the implied image metric may not preserve details important to scientists.

In the absence of a task-specific loss, we used a mixture of three common image metrics: L1 error, structural similarity (SSIM) ${ }^{19}$, and the Learned Perceptual Image Patch Similarity (LPIPS) metric $^{20}$. We empirically settled on weights of $0.2,0.5$, and 0.3 for the three metrics, respectively.

The model was trained for 600,000 steps using a batch size of eight and the Adam ${ }^{21}$ optimizer. The learning rate was initially set to $10^{-4}$ and linearly reduced to $10^{-5}$ between steps $350 \mathrm{k}$ and $550 \mathrm{k}$, and then down to $10^{-6}$ by step $600 \mathrm{k}$. We experimented with other nonlinear activation functions (relu, leaky relu, swish, tanh, sigmoid, etc.), different normalization layers (instance, layer, and channel-normalization), and with different weights for the image metrics. Visually, changing the activation function did not have a large effect, normalization layers degraded visual quality, and the loss weights only had a significant effect when extreme values were used, e.g., a weight of 1.0 for LPIPS and 0.0 for L1 and SSIM.

\section{Codecs}

We experimented with two state of the art codecs: JPEG XL (JXL) and the AV1 Image File Format (AVIF). Both are suitable for our task and outperform older image codecs such as JPEG, JPEG2000, and WebP. Either codec could be used in practice, with JPEG XL showing faster encode/decode times, while AV1 supports higher compression rates and slightly better rate-distortion performance. We believe the compression quality difference is because JPEG XL was optimized for near lossless subjective quality, which may not transfer well to the domain of EM images of brain tissue. We generated images at different compression rates by varying the 
JXL distance and AVIF $q$ parameter. We set the JXL effort option to 9 and the AVIF speed parameter to 1 . We observed that denoising led to higher relative gains in compression rates when the AVIF codec was used (see Sup. Fig. 1).

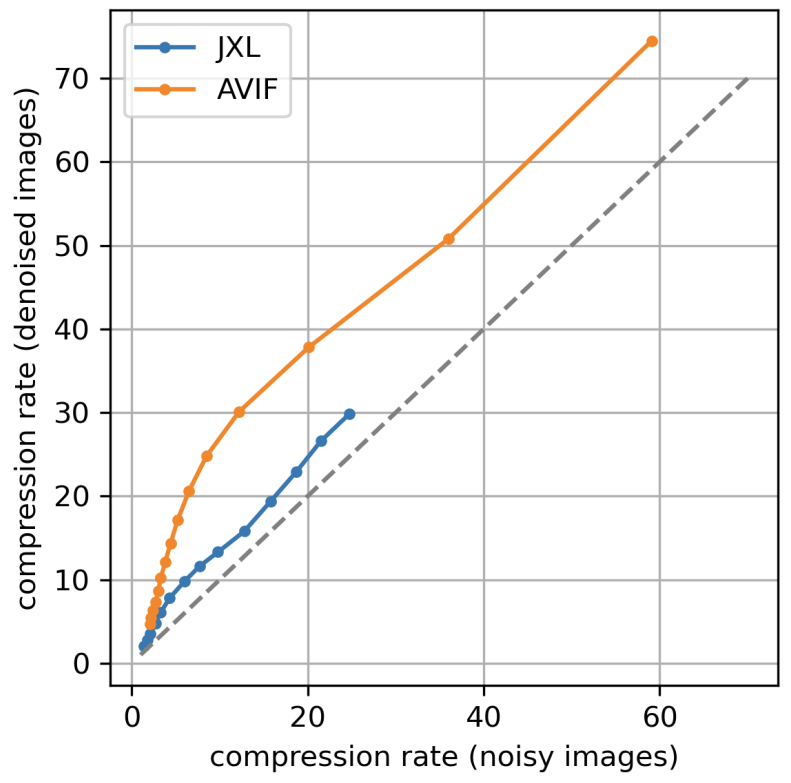

Sup. Fig. 1. Compression rate of noisy and denoised images at constant JXL distance and AVIF $q$ parameter.

\section{Segmentation and Evaluation}

We performed automated reconstruction experiments over a subset of the H01 volume ("ROI215") - a 98.3 x 98.3 x $9.9 \mu \mathrm{m}^{3}$ region within layer 2 of the cortex. For neuron segmentation we used a simplified version of the multiscale flood-filling network (FFN) pipeline $^{5,11}$. Specifically, we trained 6 separate FFN models operating on original or denoised data at $32 \times 32 \times 30 \mathrm{~nm}^{3}, 16 \times 16 \times 30 \mathrm{~nm}^{3}$ and $8 \times 8 \times 30 \mathrm{~nm}^{3}$ voxel size. We denoised the images at their original acquisition resolution of $4 \times 4 \times 30 \mathrm{~nm}^{3}$ per voxel and downsampled them with area-averaging to $8 \times 8 \times 30 \mathrm{~nm}^{3}$ voxel size. Compression, when used, was applied at $8 \times 8 \times 30 \mathrm{~nm}^{3}$ resolution, before further downsampling. The compression rates we report use the $8 \times 8 \times 30 \mathrm{~nm}^{3}$ image sizes as baseline. We used the FFN models to first build a "base segmentation" minimizing the number of false mergers, and then applied an agglomeration procedure to reduce split errors.

We computed the oversegmentation consensus ${ }^{11}$ between four input segmentations - the outputs of the $32 \times 32 \times 30 \mathrm{~nm}^{3}$ and $16 \times 16 \times 30 \mathrm{~nm}^{3}$ FFN models each executed with forward and reverse seed ordering, and filtered to retain segments comprising 10,000 voxels or larger at their respective resolution. The base segmentation was then formed by filling the remaining unsegmented voxels with the $8 \times 8 \times 30 \mathrm{~nm}^{3} \mathrm{FFN}$ model. Unlike the full $\mathrm{H} 01$ dataset, $\mathrm{ROI} 215$ does not contain any sufficiently large image irregularities (alignment problems, imaging artifacts) to require the use of FFN movement restriction or tissue masking. 
We used FFN resegmentation to agglomerate objects from the base segmentation as described previously ${ }^{11}$. We computed agglomeration scores with all three FFN models (for $32 \times 32 \times 30 \mathrm{~nm}^{3}$, $16 \times 16 \times 30 \mathrm{~nm}^{3}$ and $8 \times 8 \times 30 \mathrm{~nm}^{3}$ voxel sizes), and considered a segment pair as an edge in the agglomeration graph if the scores fulfilled the agglomeration criteria ${ }^{11}$ in the results of any of the three FFN models. For the agglomeration we only considered pairs of segments: 1) present prior to the $8 \times 8 \times 30 \mathrm{~nm}^{3}$ fill-in when using the $16 \times 16 \times 30 \mathrm{~nm}^{3}$ and $32 \times 32 \times 30 \mathrm{~nm}^{3} \mathrm{FFN}$ models; and 2) not already evaluated in step 1) when using the $8 \times 8 \times 30 \mathrm{~nm}^{3} \mathrm{FFN}$ model. Every agglomeration graph was post-processed to enforce separation between the 29 somas contained within the volume ${ }^{5}$.

We compared the $50 \mathrm{Gvx}$ FFN segmentations to a proofread sparse skeleton reconstruction of ROI215 with $0.97 \mathrm{M}$ skeleton edges covering 14,568 neurite fragments with a total path length of $282 \mathrm{~mm}$ (Sup. Fig. 2). For every segment, we classified the corresponding skeleton edges as correct, split, merged, or omitted, and from these classifications derived the edge accuracy and expected run length (ERL) metrics ${ }^{11}$ to quantify how well the automated segmentation reflected the ground truth skeletons. We randomly split the ground truth skeletons 19:1 into a testing and a tuning set. The maximum ERL achievable with the testing skeleton set was $35 \mu \mathrm{m}$.

We found that the lowest resolution $\left(32 \times 32 \times 30 \mathrm{~nm}^{3}\right)$ FFN model trained on noisy images could be applied to denoised images without loss of accuracy, but the same was not true for higher resolution models (Sup. Fig. 3). In segmentation experiments we therefore always used segmentation models exclusively with the type of images (noisy, denoised) they were trained on.

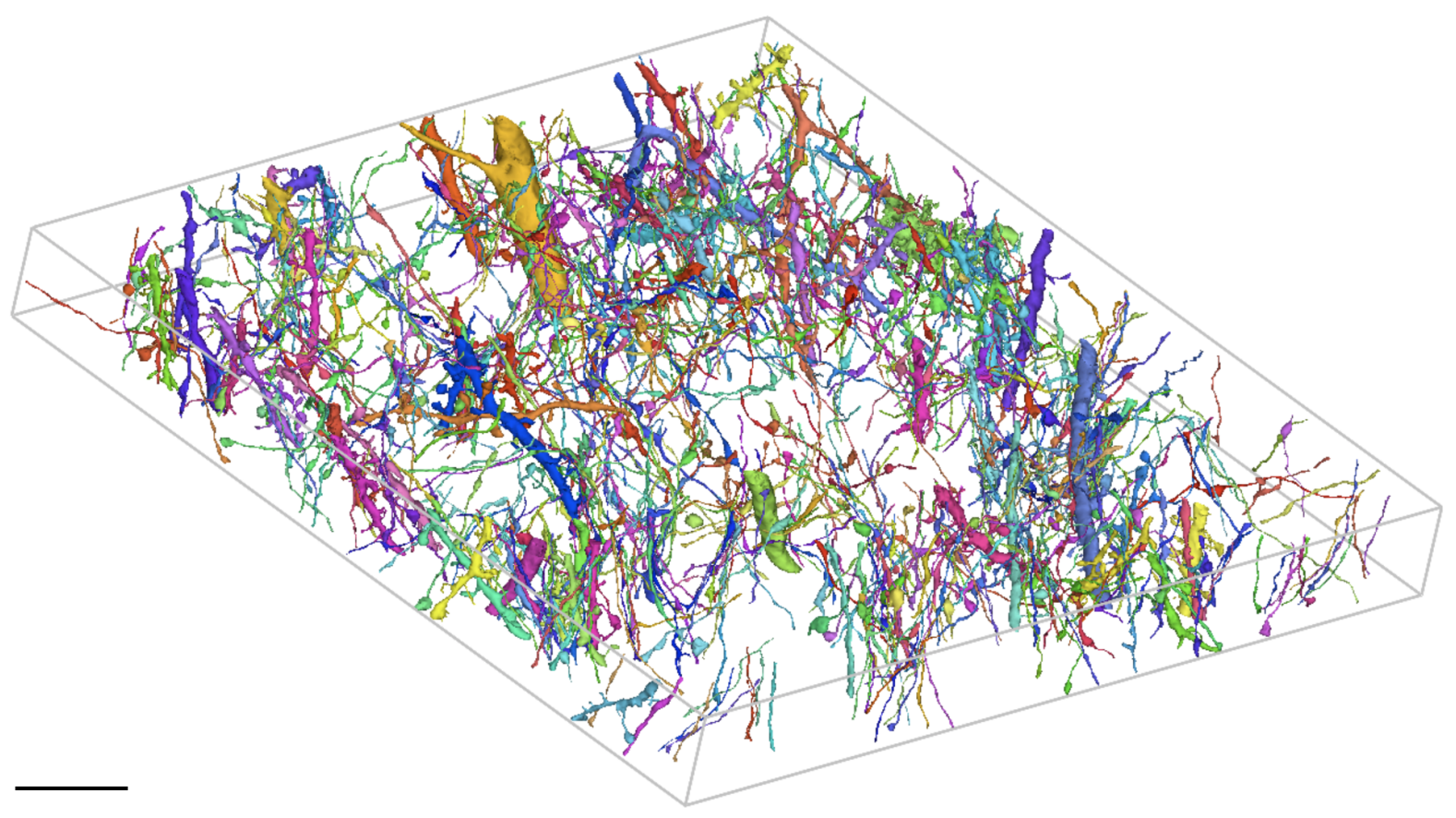

Sup. Fig. 2. Random 5\% sample of neurites in the ground truth set. Scale bar: $10 \mu \mathrm{m}$. 

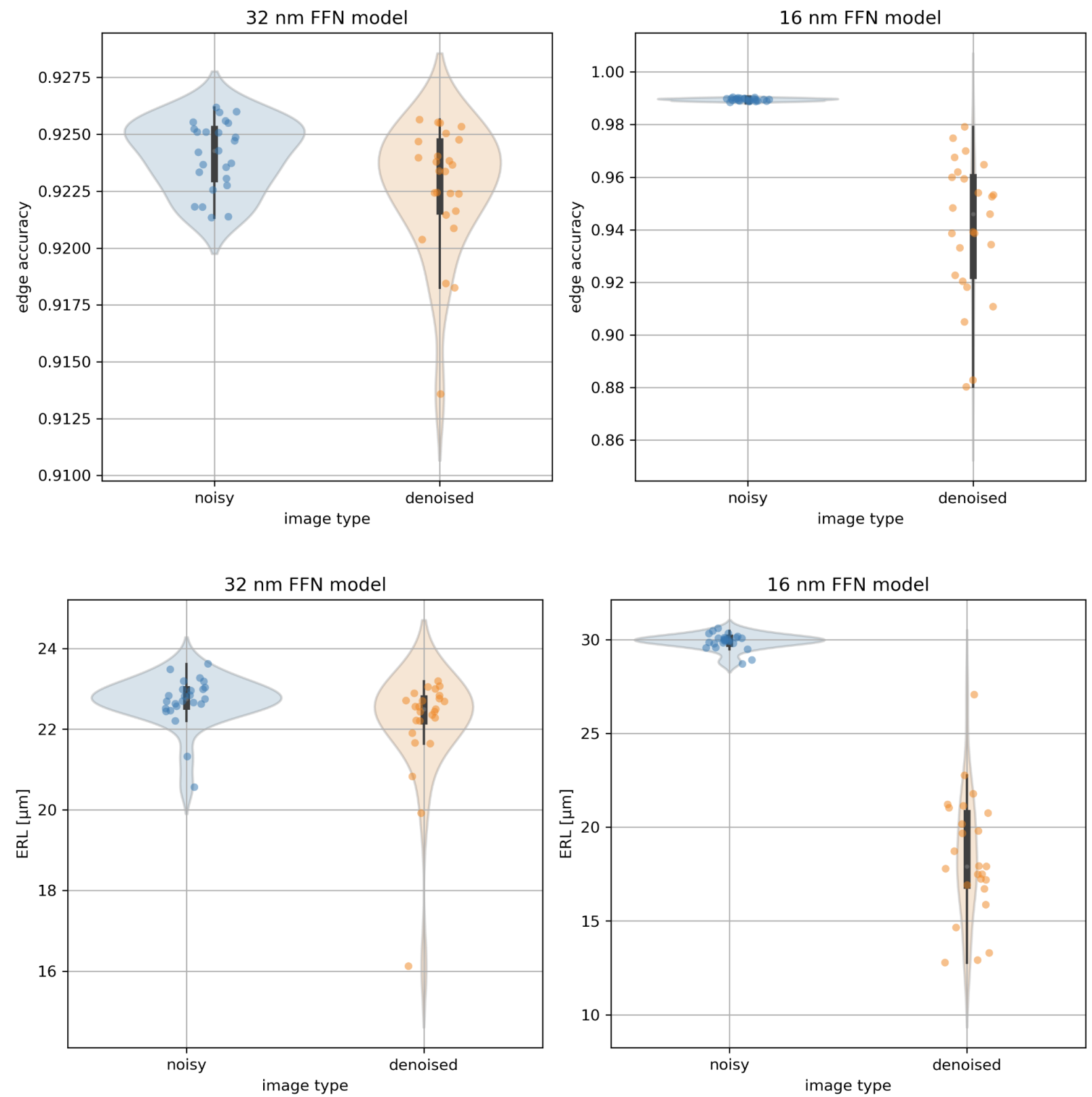

Sup. Fig. 3. Impact of image denoising on segmentation models trained on noisy data only. Dots represent weight snapshots (checkpoints) of the respective FFN model. All results in the main text were obtained by applying models to the type of data (noisy, denoised) they were trained on. 
bioRxiv preprint doi: https://doi.org/10.1101/2021.05.29.445828; this version posted May 30, 2021. The copyright holder for this preprint (which was not certified by peer review) is the author/funder, who has granted bioRxiv a license to display the preprint in perpetuity. It is made available under aCC-BY-NC-ND 4.0 International license.

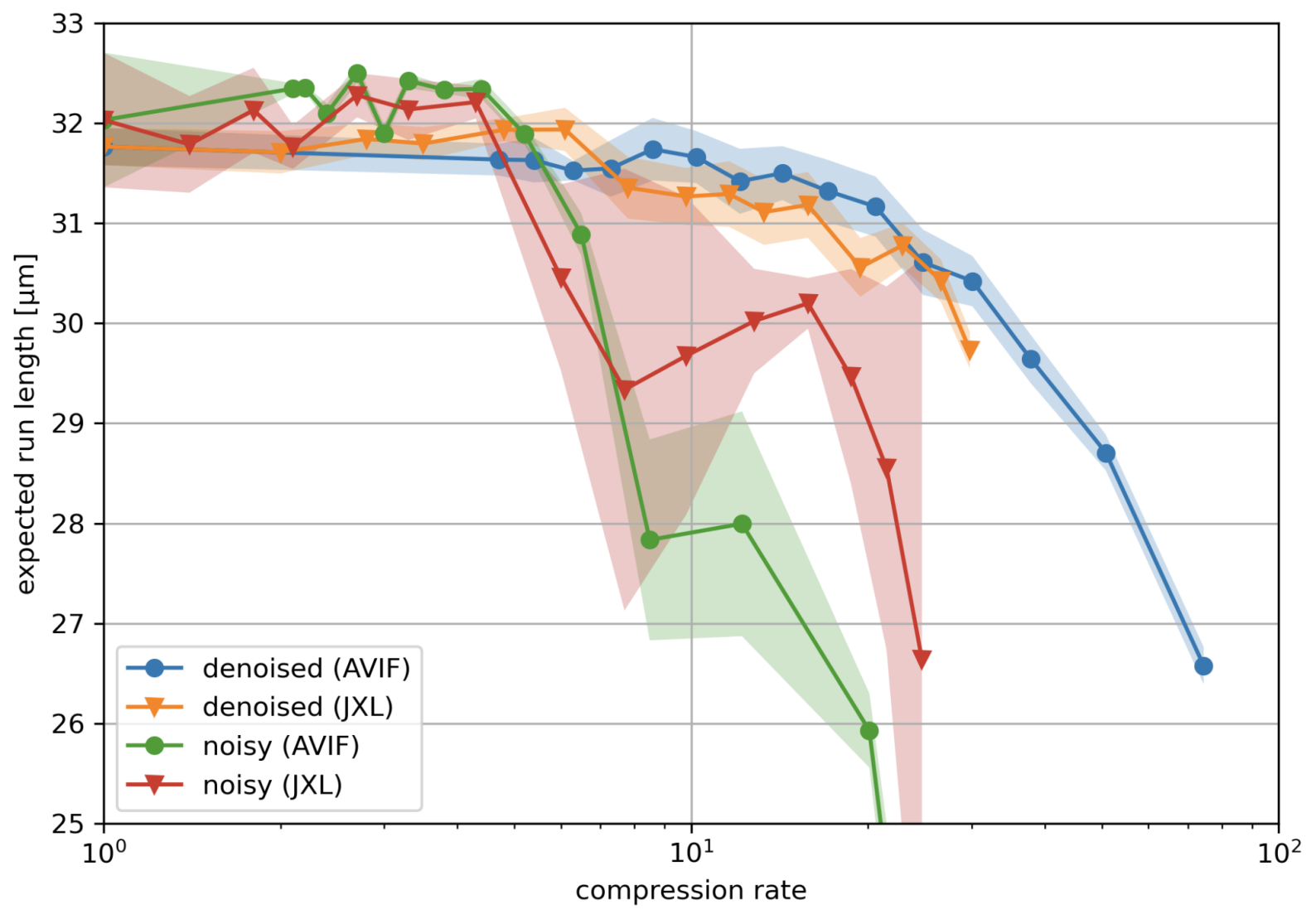

Sup. Fig. 4. Segmentation quality quantified with ERL for compressed noisy and denoised images. Note that relative quality trends closely track those seen in Fig. 3. 